\title{
ON WEAK APPROXIMATION AND CONVEXIFICATION IN WEIGHTED SPACES OF VECTOR-VALUED CONTINUOUS FUNCTIONS
}

\author{
by MAREK NAWROCKI
}

(Received 26 August, 1987)

1. Introduction. Let $X$ be a completely regular Hausdorff space. A Nachbin family of weights is a set $V$ of upper-semicontinuous positive functions on $X$ such that if $u, v \in V$ then there exists $w \in V$ and $t>0$ so that $u, v \leqslant t w$. For any Hausdorff topological vector space $E$, the weighted space $C V_{0}(X, E)$ is the space of all $E$-valued continuous functions $f$ on $X$ such that $v f$ vanishes at infinity for all $v \in V . C V_{0}(X, E)$ is equipped with the weighted topology $\omega_{V}=\omega_{V}(X, E)$ which has as a base of neighbourhoods of zero the family of all sets of the form

$$
N(v, W)=\left\{f \in C V_{0}(X, E): v f(X) \subseteq W\right\},
$$

where $v \in V$ and $W$ is a neighbourhood of zero in $E$. If $E$ is the scalar field, then the space $C V_{0}(X, E)$ is denoted by $C V_{0}(X)$. The reader is referred to $[4,6,8]$ for information on weighted spaces.

The approximation by functions with finite dimensional ranges is a very important problem while studying spaces of vector-valued functions. It is well known that if $X$ is locally compact and $E$ is locally convex, then the algebraic tensor product $C V_{0}(X) \otimes E$ is $\omega_{V}$-dense in $C V_{0}(X, E)$. $\left(C V_{0}(X) \otimes E\right.$ is the subspace of $C V_{0}(X, E)$ spanned by the functions of the form $\psi \otimes e(\psi \otimes e(x)=\psi(x) e)$, where $\psi \in C V_{0}(X)$ and $e \in E$.) In fact $C_{c}(X) \otimes E$ is dense in $C V_{0}(X, E)$, where $C_{c}(X)$ is the space of functions whose supports are compact. If $E$ is not locally convex then, though it is possible to prove $\omega_{V}$-density of $C V_{0}(X) \otimes E$ in $C V_{0}(X, E)$ making some restrictions on $X$ or $E$ (see $[3,4,9]$ ), the approximation problem is open.

In this paper we consider a "convexified" version of the approximation problem. We prove that if $X$ is locally compact then $C V_{0}(X) \otimes E$ is always weakly dense in $C V_{0}(X, E)$. This implies that $C V_{0}(X) \otimes E$ is dense in $C V_{0}(X, E)$ equipped with the locally convex topology $\omega_{V}^{c}$ associated to $\omega_{V}$ (i.e., the strongest locally convex topology on $C V_{0}(X, E)$ which is weaker than $\left.\omega_{V}\right)$. Moreover, we show that $\omega_{V}^{c}(X, E)$ coincides with the topology induced on $C V_{0}(X, E)$ by the weighted topology $\omega_{V}\left(X, E^{c}\right)$ of the space $C V_{0}\left(X, E^{c}\right)$, where $E^{c}$ is the locally convex space associated to $E$. Using this last result we obtain a representation of $\omega_{V}$-continuous linear functionals on $C V_{0}(X, E)$.

Let us note some special cases which are covered by our results.

(a) If $V=\left\{\chi_{X}\right\}$, where $\chi_{X}$ is the characteristic function of $X$, then $C V_{0}(X, E)$ is the space of all continuous functions vanishing at infinity and $\omega_{V}$ is the uniform topology. If $X$ is compact, then the weak density of $C(X) \otimes E$ in $C(X, E)$ was proved by $\mathbf{N}$. J. Kalton [2].

(b) If $V$ is the set of all non-negative upper-semicontinuous functions on $X$ which

Glasgow Math. J. 31 (1989) 59-64. 
vanish at infinity, then $C V_{0}(X, E)$ is the space of all bounded continuous functions and $\omega_{V}$ is the strict topology. This case was studied in my earlier paper [6].

It is worthwhile to note the following two examples.

(c) If $V=\left\{\chi_{K}: K\right.$ is a compact $\left.\subseteq X\right\}$, then $C V_{0}(X, E)$ is the space of all continuous functions from $X$ to $E$ and $\omega_{V}$ is the compact-open topology.

(d) If $X=\mathbb{B}^{n}, V=\{|p|: p$ is a polynomial $\}$, then $C V_{0}(X, E)$ is the space of functions rapidly decreasing at infinity.

2. Convergence with respect to submeasures and weights. Throughout this paper we will denote by $Z(X)$ the family of all zero and by $c Z(X)$ the family of all cozero subsets of a completely regular Hausdorff space $X . B(X)$ is the algebra generated by $Z(X)$ and $I(X)$ is the set of all continuous functions on $X$ with values in the interval $[0,1]$. We refer to [1] for information on zero and cozero sets.

If $f$ is a scalar or vector-valued function on $X$ and $U \in c Z(X)$, then $F \ll U$ means that there exists a $Z \in Z(X)$ such that $N(f) \subseteq Z \subseteq U$, where $N(f)=\{x: f(x) \neq 0\}$. For a subset $A$ of $X$ we write $A \ll U$ if $\chi_{A} \ll U$, where $\chi_{A}$ is the characteristic function of $A$.

Let $S(X)$ be the family of all set functions $m: c Z(X) \rightarrow[0, \infty]$ satisfying:

(S1) $m(\varnothing)=0$,

(S2) $m\left(U_{1}\right) \leqslant m\left(U_{2}\right)$ if $U_{1} \subseteq U_{2}$,

(S3) $m\left(U_{1} \cup U_{2}\right) \leqslant m\left(U_{1}\right)+m\left(U_{2}\right)$,

(S4) for every $U \in c Z(X)$ and an $\varepsilon>0$ there is a $Z \in Z(X)$ such that $Z \subseteq U$ and $m(U \backslash Z) \leqslant \varepsilon$.

It easily follows from (S1)-(S4) that the family of all subsets of $X$ satisfying

$$
\text { for every } \varepsilon>0 \text { there are } U \in c Z(X) \text { and } Z \in Z(X)
$$

such that $Z \subseteq B \subseteq U$ and $m(U \backslash Z) \leqslant \varepsilon$

is an algebra containing $Z(X)$. Therefore, $\left({ }^{*}\right)$ holds for any set $B$ belonging to $B(X)$.

The family $S(X)$ appears naturally while studying continuous linear functionals on $C V_{0}(X, E)$. Indeed, we have the following result.

LEMMA 2.1. Let $N=N(v, W)$ be an $\omega_{V}$-neighbourhood of zero in $C V_{0}(X, E)$, where $W$ is balanced. If $T$ is a linear functional on $C V_{0}(X, E)$ which is bounded on $N$, then the function $m$ defined on $c Z(X)$ by

$$
m(U)=\sup \{|T f|: f \in N, f \ll U\}
$$

belongs to $S(X)$.

Proof. Properties (S1), (S2) are obvious. We shall show that $m$ satisfies (S3).

Fix $U_{1}, U_{2} \in c Z(X), \varepsilon>0$ and $f \in N$ such that $N(f) \subseteq Z \subseteq U_{1} \cup U_{2}$, for some $Z \in Z(X)$. vf vanishes at infinity so there is a compact subset $K_{\varepsilon}$ of $X$ such that $v f\left(X \backslash K_{\varepsilon}\right) \subseteq \varepsilon W$. Let $K=Z \cap K_{\varepsilon} . U_{i}$ is a cozero set, so we can find a function $\psi_{i} \in I(X)$ such that $N\left(\psi_{i}\right)=U_{i}, i=1,2 . K$ is compact, so $\inf \left\{\left(\psi_{i} \vee \psi_{2}\right)(x): x \in K\right\}=a>0$. Let $\psi_{i}^{\prime}:=\left(\psi_{i} \vee \frac{1}{3} a \chi_{X}\right)-\frac{1}{3} a \chi_{X}, \quad i=1,2$, and $\psi_{0}^{\prime}:=\frac{2}{3} a \chi_{X}-\left(\frac{2}{3} a \chi_{X} \wedge \psi_{1} \wedge \psi_{2}\right)$. Moreover, let $\phi_{i}:=\psi_{i}^{\prime}\left(\psi_{0}^{\prime}+\psi_{1}^{\prime}+\psi_{2}^{\prime}\right)^{-1}$ for $i=0,1,2$. We define $f_{i}:=\phi_{i} f$ for $i=0,1,2$. Then 
$f=f_{0}+f_{1}+f_{2}, f_{i} \in N, f_{i} \ll U_{i}$ for $i=1,2$ and $f_{0} \in \varepsilon N$. Therefore,

$$
|T f| \leqslant\left|T f_{0}\right|+\left|T f_{1}\right|+\left|T f_{2}\right| \leqslant \varepsilon \delta+m\left(U_{1}\right)+m\left(U_{2}\right),
$$

where $\delta=\sup \{|T f|: f \in N\}$. This implies (S3).

If (S4) fails to be true then for some $\varepsilon>0$ we can find, by induction, a sequence $\left\{f_{n}\right\} \subseteq N$ such that $N\left(f_{n}\right) \cap N\left(f_{m}\right)=\varnothing$ for $n \neq m$ and $T f_{n}>\varepsilon, n, m=1,2, \ldots$ However, the functions $g_{n}:=f_{1}+\ldots+f_{n}, n \in \mathbb{N}$, belong to $N$ and $T g_{n}>\varepsilon n$. This means that $T$ is unbounded on $N$. The proof is finished.

If $m \in S(X)$ we denote by $m^{*}$ the outer measure defined on $X$ by $m$; i.e., $m^{*}(A)=\inf \{m(U): U \in c Z(X), A \ll U\}$ for $A \subseteq X$.

Let us fix a Nachbin family of weights on $X$. We denote by $\mu_{V}$ the vector topology on $C V_{0}(X, E)$ which has as a base of neighbourhoods of zero the family of all sets of the form

$$
M(m, v, W, \varepsilon)=\left\{f \in C V_{0}(X, E): m^{*}(\{x \in X: v f(x) \notin W\}) \leqslant \varepsilon\right\},
$$

where $m \in S(X), v \in V, W$ is a balanced neighbourhood of zero in $E$ and $\varepsilon>0$.

Let $\gamma_{V}$ be the strongest vector topology on $C V_{0}(X, E)$ which is weaker than $\mu_{V}$ on all $\omega_{V}$-bounded subsets of $C V_{0}(X, E)$.

LEMma 2.2. Let $T$ be such a linear functional on $C V_{0}(X, E)$ that $\lim _{\alpha} T f_{\alpha}=0$ for every net $\left\{f_{\alpha}\right\} \subseteq C V_{0}(X, E)$ which is $\omega_{V}$-bounded and $\mu_{V}$-convergent to zero. Then $T$ is $\gamma_{V}$-continuous.

Proof. It is easily seen that the weakest vector topology on $C V_{0}(X, E)$ for which $T$ is continuous is weaker than $\mu_{V}$ on every $\omega_{V}$-bounded set.

Proposition 2.3. For every Nachbin family $V$ the topology $\gamma_{V}$ is stronger than the weak topology $\sigma=\sigma\left(Y, Y^{\prime}\right)$ of the space $Y=\left(C V_{0}(X, E), \omega_{V}\right)$.

Proof. Let us fix an $\omega_{V}$-continuous linear functional $T$ on $C V_{0}(X, E)$. We can find an $\omega_{V}$-neighbourhood of zero $N=N(v, W)$ such that $W$ is balanced and $|T f| \leqslant 1$ for all $f \in N$. Using $T$ and $N$ we define $m \in S(X)$ as in Lemma 2.1. Proposition 2.3 will be proved if we show that $T$ is $\gamma_{v}$-continuous.

Let $\left\{f_{\alpha}\right\}$ be a $\omega_{V}$-bounded and $\mu_{V}$-convergent to zero net in $C V_{0}(X, E)$ (cf. Lemma 2.2). Fix $\varepsilon>0$. For every $\alpha$ there are $U_{\alpha} \in c Z(X)$ and $Z_{\alpha} \in Z(X)$ such that $A_{\alpha}:=$ $\{x: v f(x) \notin \varepsilon W\} \subseteq Z_{\alpha} \subseteq U_{\alpha}$ and $m\left(U_{\alpha}\right) \leqslant m^{*}\left(A_{\alpha}\right)+\varepsilon$. There is $\psi_{\alpha} \in I(X)$ such that $\psi_{\alpha} \ll U_{\alpha}$ and $\psi_{\alpha}\left(Z_{\alpha}\right)=\{1\}$. Let $h_{\alpha}:=\psi_{\alpha} f_{\alpha}$ and $k_{\alpha}:=\left(1-\psi_{\alpha}\right) f_{\alpha}$. The net $\left\{f_{\alpha}\right\}$ is bounded, so there is $\delta>1$ such that $\left\{f_{\alpha}\right\} \subseteq \delta N$. Thus, $\delta^{-1} h_{\alpha} \in N$ and $h_{\alpha} \ll U_{\alpha}$, so that $\left|T h_{\alpha}\right| \leqslant \delta m\left(U_{\alpha}\right) \leqslant \delta\left(m^{*}\left(A_{\alpha}\right)+\varepsilon\right)$. Moreover, $k_{\alpha} \in \varepsilon N$, and so $\left|T f_{\alpha}\right| \leqslant \delta\left(m^{*}\left(A_{\alpha}\right)+\varepsilon\right)+\varepsilon$. This implies that $\lim T f_{\alpha}=0$. The proof is finished.

3. $\gamma_{V}$ and weak approximation. Throughout the remaining part of this paper we will denote by $C V_{0}(X) \otimes_{d} E$ the subset of $C V_{0}(X, E)$ consisting of all functions of the 
form $\sum_{i=1}^{n} \psi_{i} \otimes e_{i}$, where $\psi_{i} \in C V_{0}(X), N\left(\psi_{i}\right) \cap N\left(\psi_{j}\right)=\varnothing$ if $i \neq j, e_{i} \in X, i, j=1, \ldots, n$, $n \in \mathbb{N}$.

THEOREM 3.1. Let $V$ be a Nachbin family of weights on a locally compact Hausdorff space $X$. For every balanced neighbourhood of zero $W$ in $E$ and $v \in V$ the $\gamma_{V}$-closure of $N(v, 2 W) \cap C V_{0}(X) \otimes_{d} X$ contains $N(v, W)$.

In particular, $C V_{0}(X) \otimes E$ is $\gamma_{V}$ and weakly dense in $C V_{0}(X, E)$.

Proof. Let us fix $v, W$ and $f \in N(v, W)$. The set

$$
A_{f}:=\{\psi f: \psi \in I(X), \text { supp } \psi \text { is compact }\}
$$

is $\omega_{V}$-bounded and contained in $N(v, W)$. Moreover, $f$ can be $\omega_{V}$-approximated by elements of $A_{f}$ (see [4, Theorem 3.1]). The topology $\mu_{V}$ is weaker than $\omega_{V}$, and so $\gamma_{V}$ is weaker than $\omega_{V}$ on $A_{f}$. This implies that $f$ belongs to the $\gamma_{V}$-closure of $A_{f}$. Therefore, for the proof of the theorem we may assume that $\operatorname{supp} f=: K$ is compact.

Let us fix some relatively compact neighbourhood $U$ of $K$ and let $D$ be the set of all functions of the form $\sum_{i=1}^{n} \psi_{i} \otimes f\left(x_{i}\right)$, where $\psi_{i} \in I(X), N\left(\psi_{i}\right) \subseteq U, N\left(\psi_{i}\right) \cap N\left(\psi_{j}\right)=\varnothing$ if $i \neq j, x_{i} \in U, i, j=1, \ldots, n, n \in \mathbb{N}$. The set $f(U)$ is bounded in $E$ and each function $v \in V$ being upper-semicontinuous is bounded on $U$, so $D$ is $\omega_{V}$-bounded. We shall show that $f$ can be $\mu_{V}$-approximated by elements of $D \cap N(v, 2 W)$.

Fix a $\mu_{V}$-neighbourhood of zero $M=M\left(m, v^{\prime}, W^{\prime}, \varepsilon\right)$, where $W^{\prime}$ is balanced. We may assume that $\sup \left\{v^{\prime}(x): x \in U\right\}=: a>0$. The set $f(K)$ is compact, so there exists a finite family $\left\{B_{1}, \ldots, B_{n}\right\}$ of pairwise disjoint subsets of $X$ such that $K \subseteq \bigcup_{i=1}^{n} B_{i} \subseteq U, B_{i} \in B(X)$, and $f(x)-f\left(x^{\prime}\right) \in a^{-1} W^{\prime}$ for every $x, x^{\prime} \in B_{i}, i=1, \ldots, n$. The submeasure $m$ satisfies $\left(^{*}\right)$, so we can choose cozero sets $U_{1}, \ldots, U_{n}$ and zero sets $Z_{1}, \ldots, Z_{n}$ such that $Z_{i} \subseteq B_{i} \subseteq U_{i} \subseteq U$ and $m\left(U_{i} \backslash Z_{i}\right) \leqslant \varepsilon / n, i=1, \ldots, n$. Obviously $Z_{i}$ is compact for $i=$ $1, \ldots, n$. Using the upper-semicontinuity. of $v$ we can find pairwise disjoint cozero sets $O_{1}, \ldots, O_{n}$ such that $Z_{i} \subseteq O_{i} \subseteq U_{i}$ and $\sup \left\{v(x): x \in O_{i}\right\}<2 \sup \left\{v(x): x \in Z_{i}\right\}$ for $i=$ $1, \ldots, n$. Choose $x_{i} \in Z_{i}$ satisfying $\sup \left\{v(x): x \in O_{i}\right\}<2 v\left(x_{i}\right)$ and $\psi_{i} \in I(X)$ such that $N\left(\psi_{i}\right)=O_{i}$ and $\psi_{i}\left(Z_{i}\right)=\{1\}, i=1, \ldots, n$. We define $g:=\sum_{i=1}^{n} \psi_{i} \otimes f\left(x_{i}\right)$. Then $g \in D$ and $v(x) \psi_{i}(x)<2 v\left(x_{i}\right)$ for every $x \in O_{i}, i=1, \ldots, n$. Thus $g \in D \cap N(v, 2 W)$. Moreover, $\left\{x \in X: v^{\prime}(f-g)(x) \in W^{\prime}\right\} \subseteq \bigcup_{i=1}^{n}\left\{x \in U_{i}: v^{\prime}(f-g)(x) \notin W^{\prime}\right\}$

$$
\subseteq \bigcup_{i=1}^{n}\left\{x \in U_{i}:(f-g)(x) \notin a^{-1} W^{\prime}\right\} \subseteq \bigcup_{i=1}^{n}\left(U_{i} \backslash Z_{i}\right)
$$

Therefore,

$$
m^{*}\left(\left\{x \in X: v^{\prime}(f-g)(x) \notin W^{\prime}\right\}\right) \leqslant \sum_{i=1}^{n} m\left(U_{i} \backslash Z_{i}\right) \leqslant \varepsilon,
$$


and so $f-g \in M$. The proof of the first part of this theorem is finished. The second assertion immediately follows from the first one and Proposition 2.3.

REMARK 3.2. The author does not know whether Theorem 3.1 holds if $X$ is non locally compact.

\section{The locally convex space associated with $\left(C V_{0}(X, E), \omega_{V}\right)$.}

THEOREM 4.1. Let $V$ be a Nachbin family on a locally compact Hausdorff space $X$. For every Hausdorff t.v.s $E$ the locally convex topology associated with the weighted topology $\omega_{V}(X, E)$ coincides with the topology induced on $C V_{0}(X, E)$ by the weighted topology $\omega_{V}\left(X, E^{c}\right)$ of the space $C V_{0}\left(X, E^{c}\right)$, where $E^{c}=\left(E, \tau^{c}\right)$ is the locally convex space associated with $E=(E, \tau)$.

Proof. The topology $\tau^{c}$ is weaker than $\tau$, so that $C V_{0}(X, E) \subseteq C V_{0}\left(X, E^{c}\right)$ and the inclusion mapping is continuous. Therefore, $\omega_{V}^{c}(X, E) \geqslant\left.\omega_{V}\left(X, E^{c}\right)\right|_{C V_{0}(X, E) \text {. For the }}$ proof of the converse inequality let us fix a convex weakly closed $\omega_{V}^{c}(X, E)$ neighbourhood $G$ of zero in $C V_{0}(X, E)$. We can find $v \in V$ and a balanced neighbourhood $W$ of zero in $E$ such that $\operatorname{conv} N(v, W) \subseteq G$. The set $N:=\left\{f \in C V_{0}(X, E): v f(X) \subseteq\right.$ $1 / 2 \overline{\operatorname{conv}} W\}$ is an $\left.\omega_{V}\left(X, E^{c}\right)\right|_{C v_{0}(X, E)}$-neighbourhood of zero, which is $\omega_{V}(X, E)$-closed. Therefore, $N$ is closed in the weak topology $\sigma$ of $\left(C V_{0}(X, E), \omega_{V}\right)$. Let

$$
\left.N_{d}:=N(v, \overline{\operatorname{conv}} W) \cap\left(C V_{0}(X) \otimes_{d} E\right)\right)
$$

and

$$
N_{d \infty}:=\left\{f \in C V_{0}(X) \otimes_{d} E: v f(X) \subseteq W_{\infty}\right\}
$$

where $W_{\infty}:=\bigcup_{n=1}^{\infty} W_{n}$ and $W_{n}:=2^{-n} \sum_{i=1}^{2^{n}} W$. Since $\sigma \leqslant \gamma_{V}$, Theorem 3.1 shows that

$$
N=\bar{N}^{\sigma} \subseteq \bar{N}_{d}^{\sigma}
$$

$W_{\infty}$ is $\tau$-dense in $\overline{\text { conv }} W$, and so $N_{d \infty}$ is $\omega_{V}$-dense in $N_{d}$. Consequently,

$$
N_{d \infty} \text { is weakly dense in } N_{d} \text {. }
$$

Let us observe now that

$$
N_{d \infty} \subseteq \operatorname{conv} N(v, W) \text {. }
$$

Indeed, if $f \in N_{d \infty}$ then we can find $\psi_{1}, \ldots, \psi_{k} \in C V_{0}(X)$ and $e_{1}, \ldots, e_{k} \in W_{\infty}$ such that $N\left(\psi_{i}\right) \cap N\left(\psi_{j}\right)=\varnothing$ if $i \neq j, \sup \left\{v \psi_{j}(x): x \in X\right\} \leqslant 1, i, j=1, \ldots, k$. There are $e_{i, j} \in W$, $i=1, \ldots, k, j=1, \ldots, m, m \in \mathbb{N}$, such that $e_{i}=m^{-1} \sum_{j=1}^{m} e_{i, j}$ for $i=1, \ldots, k$. Put $f_{j}:=$ $\sum_{i=1}^{k} \psi_{i} \otimes e_{i, j}, j=1, \ldots, m$. Then it is easily seen that $v f_{j}(X) \subseteq W$ and $f=m^{-1} \sum_{j=1}^{m} f_{j}$. Therefore, $f \in \operatorname{conv} N(v, W)$. 
Combining (1), (2), (3) we have $N \subseteq \bar{N}_{d}^{\sigma}=\bar{N}_{d \infty}^{\sigma} \subseteq \overline{\operatorname{conv}}^{o} N(v, W) \subseteq G$. Finally, $G$ is an $\left.\omega_{V}\left(X, E^{c}\right)\right|_{C V_{0}(X, E)}$-neighbourhood of zero. The proof is finished.

It is obvious that $\omega_{V}(X, E)$ and $\left.\omega_{V}\left(X, E^{c}\right)\right|_{C V_{0}(X, E)}$ produce the same spaces of continuous linear functionals on $C V_{0}(X, E)$. Moreover, $C V_{0}(X, E)$ is dense in $C V_{0}\left(X, E^{c}\right)$. Therefore, we can identify the dual spaces of $C V_{0}(X, E)$ and $C V_{0}\left(X, E^{c}\right)$. Indeed, the restriction mapping $\left.C V_{0}\left(X, E^{c}\right)^{\prime}{ }^{\prime} T \rightarrow T\right|_{C V_{0}(X, E)} \in C V_{0}(X, E)^{\prime}$ is an algebraic isomorphism. Therefore, using Theorem 4.1 and the known representation of the dual space of $C V_{0}(X, E)$, where $E$ is locally convex (see [8, Theorem 5.42]) we obtain the following result.

Proposition 4.2 Let $X$ be a locally compact Hausdorff space, $V$ any Nachbin family on $X$ and $E$ a t.v.s. whose topological dual separates the points of $E$. The dual space of $\left(C V_{0}(X, E), \omega_{V}\right)$ is isomorphic to

$$
V M_{b}\left(X, E^{\prime}\right) \equiv\left\{v m: v \in V, m \in M_{b}\left(X, E^{\prime}\right)\right),
$$

where $M_{b}\left(X, E^{\prime}\right)$ is the space of all $\left(E^{\prime}, \sigma\left(E^{\prime}, E\right)\right)$-valued Radon measures on $X$ with finite $p$-semivariation, for some continuous seminorm $p$ on $E$.

The reader is referred to [8] for the exact definition of $M_{b}\left(X, E^{\prime}\right)$.

\section{REFERENCES}

1. L. Gillman and M. Jerison, Rings of continuous functions, (Van Nostrand, 1960).

2. N. J. Kalton, Isomorphisms between spaces of vector-valued continuous functions, Proc. Edinburgh Math. Soc. 26 (1983), 29-48.

3. A. K. Katsaras, On the strict topology in non-locally convex setting, Math. Nachr. 102 (1981), 321-329.

4. L. A. Khan, On approximation in weighted spaces of continuous vector-valued functions, Glasgow Math. J. 29 (1987), 65-68.

5. V. Klee, Leray-Schauder theory without local convexity, Math. Ann. 141 (1960), 286-296.

6. L. Nachbin, Elements of approximation theory, (Van Nostrand, 1967).

7. M. Nawrocki, Strict dual of $C^{b}(X, E)$, Studia Math. 82 (1985), 33-38.

8. J. B. Prolla, Approximation of vector-valued functions, Mathematics Studies No. 25 (North-Holland, 1977).

9. A. H. Schuchat, Approximation of vector-valued continuous functions, Proc. Amer. Math. Soc. 31 (1972), 97-103.

INSTITUTE OF MATHEMATICS,

A. MICKIEWICZ UNIVERSITY,

MATEJKI 48/49, 60-769 Poznan,

POLAND 